\title{
Bayesian Analysis of two Censored Shifted Gompertz Mixture Distributions using Informative and Noninformative Priors
}

\author{
Tabassum Naz Sindhu \\ Department of Statistics, Quaid-i-Azam University 45320 \\ Islamabad 44000, Pakistan \\ sindhuqau@gmail.com \\ Muhammad Aslam \\ Department of Basic Sciences, Riphah International University \\ Islamabad, Pakistan \\ aslamsdqu@yahoo.com \\ Anum Shafiq \\ Department of Mathematics, Preston University, Kohat \\ Islamabad Campus, Pakistan \\ anumshafiq@ymail.com
}

\begin{abstract}
This study deals with Bayesian analysis of shifted Gompertz mixture model under type-I censored samples assuming both informative and noninformative priors. We have discussed the Bayesian estimation of parameters of shifted Gompertz mixture model under the uniform, and gamma priors assuming three loss functions. Further, some properties of the model with some graphs of the mixture density are discussed. These properties include Bayes estimators, posterior risks and reliability function under simulation scheme. Bayes estimates are obtained considering two cases: (a) when the shape parameter is known and (b) when all parameters are unknown. We analyzed some simulated sets in order to investigate the effect of prior belief, loss functions, and performance of the proposed set of estimators of the mixture model parameters.
\end{abstract}

Keywords: Censored data, Shifted Gompertz mixture distribution, Loss functions, Bayes estimators, Posterior risk, and Reliability function.

\section{Introduction}

Mixture distributions are quite popular when modeling populations containing two or more subgroups. These probability models represent non-homogenous behavoiurs and may be utilized in manufacturing and non-manufacturing applications for the characteristic(s) of interest that exhibit mixture patterns. These types of mixtures offer a more valuable analysis that leads to more meaningful results. It is important to properly model the variable of interest by using simple or mixture models and to estimate their parameters with the assistance of accessible information. The estimation of parameters may be carried out using classical and Bayesian methods of estimation. Gosh and Ebrahimi (2001) have been studied the Bayesian analysis of the mixing function in a mixture of two exponential distributions. Sultan et al. (2007) investigated the properties of the two component mixture of inverse Weibull distribution under classical approach. Saleem and Aslam (2008) presented a comparison study of the maximum likelihood estimates with the Bayes estimates assuming the uniform and the Jeffreys priors for the parameters of the Rayleigh mixture. Saleem et al. (2010) considered the Bayesian 
analysis of the mixture of Power function distribution using the complete and the censored sample.

Sindhu et al. (2014) has considered the Bayesian inference for mixture Burr type II distribution under type-I censoring.

In life testing experiments, it often occurs that experimenter may not be in a position to observe the life times of all items put on test because of time limitations and other restrictions on the data collection. Type-I and Type-II censoring schemes are the two most common censoring schemes. In Type-I censoring scheme, the experiment pursue up to a predetermined fixed time $T$ but the number of failures is random, whereas in Type-II censoring scheme, the experimental time is random but the number of failures is preselected, $r$. Sindhu et al. (2016) have studied the cumulative charting technique based on simple and the mixture of Rayleigh models. Sindhu et al. (2016) have considered a new methodology for Bayesian analysis of mixture models under doubly censored samples.

The modeling and forecasting of the diffusion of modernization has been a topic of increasing research interest in marketing and other fields of life. A good number of models on the acceptance and diffusion of new products and technologies have been presented over time, since the basic article of Bass (1969). Later models have integrated greater adaptability modifying and extending the Bass model in different ways. The model of Bass has been one of the main references in models of adopting timing of innovations. From this model, Bemmaor (1999) formulated a derivation for which the individual-level model of adopting timing of a new product is randomly distributed according to the shifted Gompertz distribution. Some statistical properties of the shifted Gompertz distribution are shown in Bemmaor (1999).

Bemmaor and Lee (2002) studied that the Bass model results from the mixing of shifted Gompertz and exponential distributions. More recently, Jimenez and Jodra (2009) have contributed to the study of this distribution, providing explicit expressions for the expectation and variance, deriving a closed-form expression for the quantile function and considering the limit distributions of extreme order statistics.

Gompertz distribution is used as a survival model in reliability and survival analysis. It has an increasing hazard rate for the life of the systems. This distribution does not seem to have received enough attention. Saracoglu et al. (2009) compared the maximum likelihood, uniformly minimum variance unbiased and Bayes estimators for the parameter of the Gompertz distribution. Ismail (2010) considered the Gompertz distribution as a lifetime model for applying the Bayesian approach to the estimation problem in the case of step stress partially accelerated life tests with two stress levels and type-I censoring. Ismail (2011) discussed the point and interval estimations of a twoparameters Gompertz distribution under partially accelerated life tests with Type-II censoring. Kiani et al. (2012) studied the performance of the Gompertz model with timedependent covariate in the presence of right censored data. Torres (2014) proposed nonlinear least squares procedures for estimating the parameters of the shifted Gompertz distribution. Sindhu et al. (2014) studied Bayesian analysis of the shape parameter of the mixture of Burr type $\mathrm{X}$ distribution using the censored data. 
Form the literature survey; it is obvious that no attempt has been made so far to analyze the study of shifted Gompertz mixture distribution. Also the shifted Gompertz distribution does not seem to have been studied in the statistical literature under the Bayesian framework. Motivated by the study of shifted Gompertz distribution, thus the aim of present article is to investigate the prominent features of shifted Gompertz mixture distribution under Bayesian structure. The rest of the paper is organized as follows. In Section 2, we define the mixture model, and likelihood function of shifted Gompertz mixture distribution. Section 3, deals with Bayesian estimation of the parameters when shape parameter is known, which includes the posterior distribution, Bayes estimators and posterior risks under different loss functions. A Simulation study and comparison of the estimates are given in Section 4. The Bayesian estimation of the parameters and reliability function when all parameters are unknown is considered in Section 5. Some concluding remarks are presented in Section 6.

\section{Mixture model and likelihood function}

A finite mixture model with 2-component densities of specified parametric form and unknown mixing proportion $\left(p_{i}\right)$ is defined as:

$f(x ; \Theta)=p_{1} f_{1}\left(x_{1 j}, \Theta_{1}\right)+p_{2} f_{2}\left(x_{2 j}, \Theta_{2}\right), \sum_{i=1}^{2} p_{i}=1$,

where $\Theta=\left(p_{1}, \lambda_{1}, \lambda_{2}, \eta_{1}, \eta_{2}\right), p_{2}=1-p_{1}$ and $\Theta_{i}=\left(\lambda_{i}, \eta_{i}\right), i=1,2$.

The random variable $\mathrm{X}$ has the shifted Gompertz distribution with the p.d.f is assumed for two components of the mixture:

$$
f_{i}\left(x \mid \lambda_{i}, \eta_{i}\right)=\lambda_{i} \exp \left(-\lambda_{i} x\right) \exp \left\{-\eta_{i} \exp \left(-\lambda_{i} x\right)\right\}\left\{1+\eta_{i}\left(1-\exp \left(-\lambda_{i} x\right)\right)\right\}, x>0, \lambda_{i}, \eta_{i}>0, i=1,2 .
$$

Where $\eta_{i}>0$ is a shape parameter and $\lambda_{i}>0$ is a scale parameter of the density function of the $i^{\text {th }}$ component. Then the distribution of the corresponding mixture distribution is:

$$
\begin{aligned}
& F(T ; \Theta)=p_{1} F_{1}\left(T ; \Theta_{1}\right)+p_{2} F_{2}\left(T ; \Theta_{2}\right), \\
& F(T ; \Theta)=p_{1}\left\{1-\exp \left(-\lambda_{1} T\right)\right\} \exp \left\{-\eta_{1} \exp \left(-\lambda_{1} T\right)\right\}+p_{2}\left\{1-\exp \left(-\lambda_{2} T\right)\right\} \exp \left\{-\eta_{2} \exp \left(-\lambda_{2} T\right)\right\} .
\end{aligned}
$$

Graphical representations for different selected parametric values for the mixture model are shown in Fig. 1.

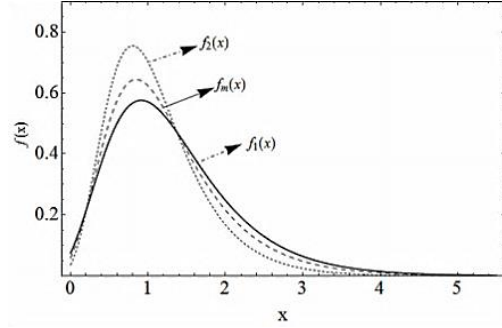

(i)

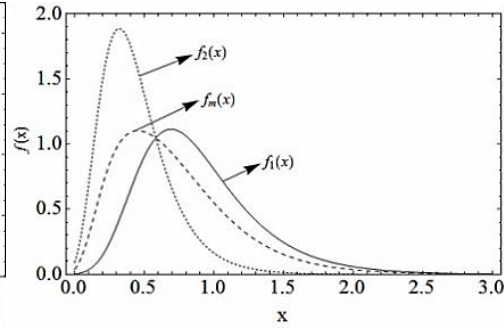

(ii)

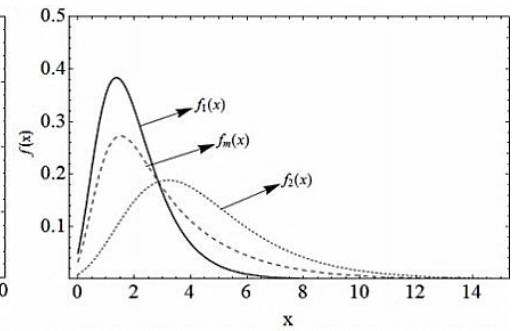

(iii) 


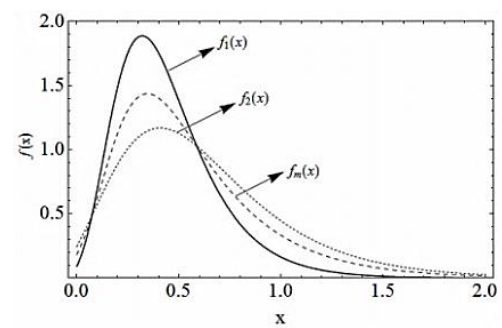

(iv)

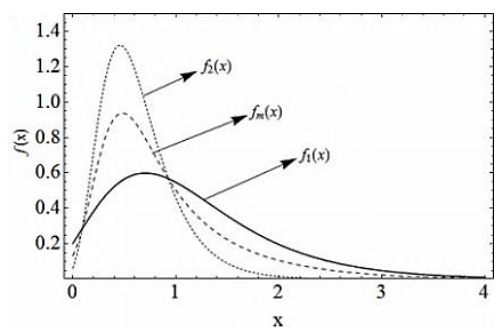

$(v)$

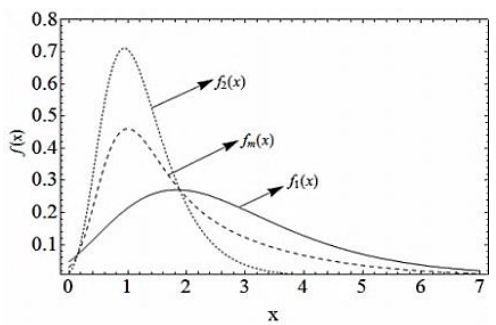

(vi)

Fig. 1: Density function components and their mixtures $\left(p_{1}, \lambda_{1}, \lambda_{2}, \eta_{1}, \eta_{2}\right):(i)(0.6,3,5,7,4),(i i)(0.6$, 1, 0.5, 3, 4), (iii) $(0.4,5,3,4,2.5),($ iv) $(0.3,2.5,1.5,6,4),(v)(0.5,1.5,3.5,2,4),(v i)(0.5,0.7,1.9,2.7,5)$

The mean median and mode of the two-component mixture of the shifted Gompertz distribution are obtained and shown in Table 1.

Table 1: Mean Median and Mode for the two-component mixture of the Gompertz distribution.

\begin{tabular}{|c|c|c|c|c|c|c|c|}
\hline$\left(p_{1}, \lambda_{1}, \lambda_{2}, \eta_{1}, \eta_{2}\right)$ & Mean & Median & Mode & $\left(p_{1}, \lambda_{1}, \lambda_{2}, \eta_{1}, \eta_{2}\right)$ & Mean & Median & Mode \\
\hline $0.1,1,4,3,6$ & 0.77107 & 0.61332 & 0.48723 & $0.1,3,0.5,8,1.5$ & 2.97383 & 2.40250 & 0.79406 \\
\hline $0.2,1,4,3,6$ & 0.90824 & 0.65551 & 0.48935 & $0.2,3,0.5,8,1.5$ & 2.74643 & 2.07476 & 0.75887 \\
\hline $0.3,1,4,3,6$ & 1.04541 & 0.71106 & 0.49202 & $0.3,3,0.5,8,1.5$ & 2.51902 & 1.72566 & 0.74754 \\
\hline $0.4,1,4,3,6$ & 1.18258 & 0.78786 & 0.49562 & $0.4,3,0.5,8,1.5$ & 2.29162 & 1.42731 & 0.74193 \\
\hline $0.5,1,4,3,6$ & 1.31976 & 0.89912 & 0.50074 & $0.5,3,0.5,8,1.5$ & 2.06422 & 1.22649 & 0.73858 \\
\hline $0.6,1,4,3,6$ & 1.45693 & 1.05924 & 0.50863 & $0.6,3,0.5,8,1.5$ & 1.83682 & 1.09719 & 0.73635 \\
\hline $0.7,1,4,3,6$ & 1.59410 & 1.25743 & 0.52242 & $0.7,3,0.5,8,1.5$ & 1.60492 & 1.00890 & 0.734761 \\
\hline
\end{tabular}

The median and mode of the two components mixture of the shifted Gompertz distribution are found by solving the nonlinear equation with respect to $x$.

$$
\begin{aligned}
& \exp \left\{-\eta_{1} \exp \left(-\lambda_{1} x\right)-\eta_{2} \exp \left(-\lambda_{2} x\right)-4 x\left(\lambda_{1}+\lambda_{2}\right)\right\} \\
& \times\left\{\begin{array}{l}
p_{1} \lambda_{1}^{3}\left(-\eta_{1}^{3}+\left(1+\eta_{1}\right) \exp \left(3 \lambda_{1} x\right)+\eta_{1}{ }^{2}\left(6+\eta_{1}\right) \exp \left(\lambda_{1} x\right)-\eta_{1}\left(7+3 \eta_{1}\right) \exp \left(2 \lambda_{1} x\right)\right) \exp \left(\eta_{2} \exp \left(-\lambda_{2} x\right)+4 x \lambda_{2}\right) \\
+p_{2} \lambda_{2}{ }^{3}\left(-\eta_{2}{ }^{3}+\left(1+\eta_{2}\right) \exp \left(3 \lambda_{2} x\right)+\eta_{2}{ }^{2}\left(6+\eta_{2}\right) \exp \left(\lambda_{2} x\right)-\eta_{2}\left(7+3 \eta_{1}\right) \exp \left(2 \lambda_{2} x\right)\right) \exp \left(\eta_{1} \exp \left(-\lambda_{1} x\right)+4 x \lambda_{1}\right)
\end{array}\right\}=0 .
\end{aligned}
$$

and

$p_{1}\left\{1-\exp \left(-\lambda_{1} x\right)\right\} \exp \left\{-\eta_{1} \exp \left(-\lambda_{1} x\right)\right\}+\left(1-p_{1}\right)\left\{1-\exp \left(-\lambda_{2} x\right)\right\} \exp \left\{-\eta_{2} \exp \left(-\lambda_{2} x\right)\right\}=0.5$

The Parametric values $\lambda_{1}, \lambda_{2}, \eta_{1}, \eta_{2}$ and $p_{1}$, in Table 1 are chosen to show the increasing and decreasing order of mean, median and mode by the variation in the values of the mixing proportion $p_{1}$. 
Suppose the $n$ units from the above cited mixture model is used in life testing experiment with a fixed test termination time $T$. After the test has been performed, it is observed that out of $n$ units, $r$ units have failed till the test termination time $T$, while the remaining $n-r$ units are still working As the sampling scheme proposed by Mendenhall and Hader (1958), in many real life situation only the failed items can be identified as the members of the first and the second subpopulation respectively. Here it is clear that $r=r_{1}+r_{2}$ and the remaining $n-r$ units that are still functioning provide no information about the population to which they belong. Let xij be defined as the failure time of the jth unit from the $\mathrm{i}$ th subpopulation, where $\mathrm{j}=1,2, \ldots, \mathrm{ri}, \mathrm{i}=1,2 . \quad 0<x_{1 j}, x_{2 j} \leq T$. Then the likelihood function has the following form:

$$
\begin{aligned}
L(\Theta \mid \mathrm{x}) & \propto\left\{\prod_{j=1}^{r_{1}} p_{1} f_{1}\left(x_{1 j}\right)\right\}\left\{\prod_{j=1}^{r_{2}} p_{2} f_{2}\left(x_{2 j}\right)\right\}\{1-F(T)\}^{n-r} \\
L(\Theta \mid \mathrm{x}) \propto & \sum_{k_{1}=0}^{n-r} \sum_{k_{2}=0}^{k_{1}}\left(\begin{array}{c}
n-r \\
k_{1}
\end{array}\right)\left(\begin{array}{l}
k_{1} \\
k_{2}
\end{array}\right)(-1)^{k_{1}} p_{1}^{r_{1}+k_{1}-k_{2}} \lambda_{1}^{r_{1}} \exp \left(-\lambda_{1} \sum_{j=1}^{n} x_{1 j}\right) \exp \left\{-\eta_{1}\left(\sum_{j=1}^{n} \exp \left(-\lambda_{1} x_{1 j}\right)+\left(k_{1}-k_{2}\right) \exp \left(-\lambda_{1} T\right)\right)\right\} \\
& \times \exp \left\{\sum_{j=1}^{n} \ln \left(1+\eta_{1}\left(1-\exp \left(-\lambda_{1} x_{1 j}\right)\right)\right)+\left(k_{1}-k_{2}\right)\left\{\ln \left(1-\exp \left(-\lambda_{1} T\right)\right)\right\}\right\} p_{2}^{r_{2}+k_{2}} \lambda_{2}^{r_{2}} \exp \left(-\lambda_{2} \sum_{j=1}^{2} x_{2 j}\right) \\
& \times \exp \left\{-\eta_{2}\left(\sum_{j=1}^{2} \exp \left(-\lambda_{2} x_{2 j}\right)+k_{2} \exp \left(-\lambda_{2} T\right)\right)\right\} \exp \left\{\sum_{j=1}^{2} \ln \left(1+\eta_{2}\left(1-\exp \left(-\lambda_{2} x_{2 j}\right)\right)\right)+k_{2}\left\{\ln \left(1-\exp \left(-\lambda_{2} T\right)\right)\right\}\right\} .
\end{aligned}
$$

For the simplicity, taking $\Psi_{1}=\sum_{j=1}^{\eta} \ln \left(1+\eta_{1}\left(1-\exp \left(-\lambda_{1} x_{1 j}\right)\right)\right)+\left(k_{1}-k_{2}\right)\left\{\ln \left(1-\exp \left(-\lambda_{1} T\right)\right)\right\}$ and $\Psi_{2}=\sum_{j=1}^{2} \ln \left(1+\eta_{2}\left(1-\exp \left(-\lambda_{2} x_{2 j}\right)\right)\right)+k_{2}\left\{\ln \left(1-\exp \left(-\lambda_{2} T\right)\right)\right\}$

Hence (3) can be simplified to:

$$
\begin{aligned}
L(\Theta \mid \mathrm{x}) \propto & \sum_{k_{1}=0}^{n-r} \sum_{k_{2}=0}^{k_{1}}\left(\begin{array}{c}
n-r \\
k_{1}
\end{array}\right)\left(\begin{array}{c}
k_{1} \\
k_{2}
\end{array}\right)(-1)^{k_{1}} p_{1}^{r_{1}+k_{1}-k_{2}} \lambda_{1}^{r_{1}} \exp \left\{-\lambda_{1} \sum_{j=1}^{n} x_{1 j}-\eta_{1}\left(\sum_{j=1}^{n} \exp \left(-\lambda_{1} x_{1 j}\right)+\left(k_{1}-k_{2}\right) \exp \left(-\lambda_{1} T\right)\right)+\Psi_{1}\right\} \\
& \times p_{2}^{r_{2}+k_{2}} \lambda_{2}^{r_{2}} \exp \left\{-\lambda_{2} \sum_{j=1}^{2} x_{2 j}-\eta_{2}\left(\sum_{j=1}^{n^{2}} \exp \left(-\lambda_{2} x_{2 j}\right)+k_{2} \exp \left(-\lambda_{2} T\right)\right)+\Psi_{2}\right\} .
\end{aligned}
$$

\section{Bayesian estimation of parameters (case a: shape parameter known)}

In this section, we discuss prior distributions for unknown parameters, loss functions and Bayes estimators and their posterior risks. 


\subsection{Bayesian estimation using gamma prior}

The Bayesian analysis requires the choice of suitable priors for the unknown parameters in addition to the experimental data. There is no well-defined way in which one can say that one prior is better than other see Arnold and Press (1983). The main thing in this bond is the relationship between the prior distribution and the loss function. The mixture model under consideration has two shape parameters, two scale parameters and one mixing proportion parameter. We consider both the informative and noninformative priors and observed the results. First, we assume that scale parameter $\lambda_{i}, i=1,2$, has independent gamma prior with the shape and scale parameters as $a$ and $b$ respectively, $g\left(\lambda_{i} \mid a_{i}, b_{i}\right) \propto \lambda_{i}^{a_{i}-1} \exp \left(-b_{i} \lambda_{i}\right)$ and uniform prior for $p_{1}$. By combining the likelihood function given in (3) leads towards the following joint posterior distribution of $\lambda_{i}, p_{1}$ as:

$$
\begin{aligned}
& g(\Omega \mid \mathrm{x})=\frac{\sum_{k_{1}=0}^{n-r} \sum_{k_{2}=0}^{k_{1}}\left(\begin{array}{c}
n-r \\
k_{1}
\end{array}\right)\left(\begin{array}{c}
k_{1} \\
k_{2}
\end{array}\right)(-1)^{k_{1}} p_{1}^{r_{1}+k_{1}-k_{2}} p_{2}^{r_{2}+k_{2}} \lambda_{i}^{r_{i}+a_{i}-1} \exp \left\{-\lambda_{i}\left(b_{i}+\sum_{j=1}^{n} x_{i j}\right)\right\} \exp \left\{\Psi_{i}-\eta_{i}\left(\Upsilon_{\mathrm{i}}\right)\right\}}{\sum_{k_{1}=0}^{n-r} \sum_{k_{2}=0}^{k_{1}}\left(\begin{array}{c}
n-r \\
k_{1}
\end{array}\right)\left(\begin{array}{c}
k_{1} \\
k_{2}
\end{array}\right)(-1)^{k_{1}} B\left(r_{1}+k_{1}-k_{2}+1, r_{2}+k_{2}+1\right) \iint_{0}^{\infty} \int_{0}^{\infty} \lambda_{i}^{r_{i}+a_{i}-1} \exp \left\{-\lambda_{i}\left(b_{i}+\sum_{j=1}^{n} x_{i j}\right)\right\} \exp \left\{\Psi_{i}-\eta_{i}\left(\Upsilon_{\mathrm{i}}\right)\right\} d \lambda_{i}}, \\
& \text { where } \Omega=\left(\lambda_{1}, \lambda_{2}, p_{1}\right), \Upsilon_{1}=\sum_{j=1}^{n} \exp \left(-\lambda_{1} x_{1 j}\right)+\left(k_{1}-k_{2}\right) \exp \left(-\lambda_{1} T\right), \text { and } \Upsilon_{2}=\sum_{j=1}^{2} \exp \left(-\lambda_{2} x_{2 j}\right)+k_{2} \exp \left(-\lambda_{2} T\right) .
\end{aligned}
$$

The Marginal distribution of $\lambda_{1}$ is simply the probability distribution of $\lambda_{1}$ that neglects other nuisance information about $\lambda_{2}$ and $p_{1}$, which is obtained by integrating the joint probability distribution with respect to other parameters as:

$$
\begin{aligned}
& \sum_{k_{1}=0}^{n-r} \sum_{k_{2}=0}^{k_{1}}\left(\begin{array}{c}
n-r \\
k_{1}
\end{array}\right)\left(\begin{array}{l}
k_{1} \\
k_{2}
\end{array}\right)(-1)^{k_{1}} B\left(r_{1}+k_{1}-k_{2}+1, r_{2}+k_{2}+1\right) \lambda_{1}^{r_{1}+a_{1}-1} \exp \left\{-\lambda_{1}\left(b_{1}+\sum_{j=1}^{n} x_{1 j}\right)\right\} \exp \left\{\Psi_{1}-\eta_{1}\left(\Upsilon_{1}\right)\right\} \\
& p\left(\lambda_{1} \mid \mathrm{x}\right)= \int_{0}^{\infty} \lambda_{2}^{r_{2}+a_{1}-1} \exp \left\{-\lambda_{2}\left(b_{2}+\sum_{j=1}^{n_{2}} x_{2 j}\right)\right\} \exp \left\{\Psi_{2}-\eta_{2}\left(\Upsilon_{2}\right)\right\} d \lambda_{2} \\
& \sum_{k_{1}=0}^{n-r} \sum_{k_{2}=0}^{k_{1}}\left(\begin{array}{c}
n-r \\
k_{1}
\end{array}\right)\left(\begin{array}{c}
k_{1} \\
k_{2}
\end{array}\right)(-1)^{k_{1}} B\left(r_{1}+k_{1}-k_{2}+1, r_{2}+k_{2}+1\right) \int_{0}^{\infty} \int_{0}^{\infty} \lambda_{i}^{r_{i}+a_{i}-1} \exp \left\{-\lambda_{i}\left(b_{i}+\sum_{j=1}^{n} x_{i j}\right)\right\} \exp \left\{\Psi_{i}-\eta_{i}\left(\Upsilon_{i}\right)\right\} d \lambda_{i}
\end{aligned}
$$

Similarly, the marginal posterior distribution of $\lambda_{2}$ and $p_{1}$ are derived as:

$$
\begin{gathered}
\sum_{k_{1}=0}^{n-r} \sum_{k_{2}=0}^{k_{1}}\left(\begin{array}{c}
n-r \\
k_{1}
\end{array}\right)\left(\begin{array}{l}
k_{1} \\
k_{2}
\end{array}\right)(-1)^{k_{1}} B\left(r_{1}+k_{1}-k_{2}+1, r_{2}+k_{2}+1\right) \lambda_{2}^{r_{2}+a_{1}-1} \exp \left\{-\lambda_{2}\left(b_{2}+\sum_{j=1}^{n} x_{2 j}\right)\right\} \exp \left\{\Psi_{2}-\eta_{2}\left(\Upsilon_{2}\right)\right\} \\
p\left(\lambda_{2} \mid \mathrm{x}\right)=\frac{\int_{0}^{\infty} \lambda_{1}^{r_{1}+a_{1}-1} \exp \left\{-\lambda_{1}\left(b_{1}+\sum_{j=1}^{n} x_{1 j}\right)\right\} \exp \left\{\Psi_{1}-\eta_{1}\left(\Upsilon_{1}\right)\right\} d \lambda_{1}}{\sum_{k_{1}=0}^{n-r} \sum_{k_{2}=0}^{k_{1}}\left(\begin{array}{c}
n-r \\
k_{1}
\end{array}\right)\left(\begin{array}{c}
k_{1} \\
k_{2}
\end{array}\right)(-1)^{k_{1}} B\left(r_{1}+k_{1}-k_{2}+1, r_{2}+k_{2}+1\right) \iint_{0}^{\infty} \int_{0}^{\infty} \lambda_{i}^{r_{i}+a_{i}-1} \exp \left\{-\lambda_{i}\left(b_{i}+\sum_{j=1}^{n} x_{i j}\right)\right\} \exp \left\{\Psi_{i}-\eta_{i}\left(\Upsilon_{i}\right)\right\} d \lambda_{i}} . \\
p(w \mid \mathrm{x})=\frac{\sum_{k_{1}=0}^{n-r} \sum_{k_{2}=0}^{k_{1}}\left(\begin{array}{c}
n-r \\
k_{1}
\end{array}\right)\left(\begin{array}{c}
k_{1} \\
k_{2}
\end{array}\right)(-1)^{k_{1}} p_{1}^{r_{1}+k_{1}-k_{2}} p_{2}^{r_{2}+k_{2}} \int_{0}^{\infty} \int_{0}^{\infty} \lambda_{i}^{r_{i}+a_{i}-1} \exp \left\{-\lambda_{i}\left(b_{i}+\sum_{j=1}^{n} x_{i j}\right)\right\} \exp \left\{\Psi_{i}-\eta_{i}\left(\Upsilon_{i}\right)\right\} d \lambda_{i}}{\sum_{k_{1}=0}^{n-r} \sum_{k_{2}=0}^{k_{1}}\left(\begin{array}{c}
n-r \\
k_{1}
\end{array}\right)\left(\begin{array}{c}
k_{1} \\
k_{2}
\end{array}\right)(-1)^{k_{1}} B\left(r_{1}+k_{1}-k_{2}+1, r_{2}+k_{2}+1\right) \int_{0}^{\infty} \int_{0}^{\infty} \lambda_{i}^{r_{i}+a_{i}-1} \exp \left\{-\lambda_{i}\left(b_{i}+\sum_{j=1}^{n} x_{i j}\right)\right\} \exp \left\{\Psi_{i}-\eta_{i}\left(\Upsilon_{i}\right)\right\} d \lambda_{i}} .
\end{gathered}
$$




\subsection{Bayesian estimation of the mixture model assuming the noninformative prior}

The noninformative priors are significant part of Bayesian tool kit. The noninformative priors have a least effect on the ultimate inference comparative to the data. Bernardo (1979) contended that a noninformative prior should be considered as a reference prior, i.e., a prior that is favourable for use as a standard when scrutinize statistical data. The most common example of noninformative prior is uniform prior and is employed when no conventional prior information is available. The uniform prior for the unknown parameter $\lambda_{i}$ can be written as $\lambda_{i} \square \operatorname{Uniform}(0, \infty), i=1,2$. We suppose a priori that $\left(\lambda_{i}, p_{1}\right)$ are independent and also assume that $p_{1} \square \operatorname{Uniform}(0,1)$. Thus the joint prior distribution of $\left(\lambda_{i}, p_{1}\right)$ is $p\left(\lambda_{i}, p_{1}\right) \propto k$. By merging the likelihood function given in (3) with uniform prior information, we obtained the joint posterior distribution as:

$p(\Omega \mid \mathrm{x}) \propto \sum_{k_{1}=0}^{n-r} \sum_{k_{2}=0}^{k_{1}}\left(\begin{array}{c}n-r \\ k_{1}\end{array}\right)\left(\begin{array}{l}k_{1} \\ k_{2}\end{array}\right)(-1)^{k_{1}} p_{1}^{r_{1}+k_{1}-k_{2}} p_{2}^{r_{2}+k_{2}} \lambda_{i}^{r_{i}} \exp \left\{-\lambda_{i} \sum_{j=1}^{\eta_{i}} x_{i j}\right\} \exp \left\{\Psi_{i}-\eta_{i}\left(\Upsilon_{\mathrm{i}}\right)\right\}$,

where $\Omega, \Upsilon_{i}$ and $\Psi_{i}$ are already defined.

Marginal distributions of $\lambda_{i}$ and $p_{1}$ can be obtained by nuisance parameters. For space restriction, we do not present the expression for the marginal distributions under noninformative priors.

\subsection{Bayesian estimators under different loss functions}

In order to select a best decision in decision theory, a suitable loss function must be specified. The preference of loss function is a difficult job, and its selection is often formed for the reasons of mathematical convenience without any particular decision problem of ongoing interest excluding cost effect. As in the risk analysis, the potentiality of undesired event and its consequences both are explored. This potentiality is usually measured by failure rate. The Bayesian approach is extensively applied to failure rate. In disastrous outcomes, it can be terrible to underestimate the potentiality of an event rather than to overestimate. This is significant when the risk level is the basis of risk reducing initiative, either by reducing the potentiality or the consequences. An inappropriately low estimate of the risk level can lead to the lack of necessary steps to reduce the risk level. Hence, it is unreasonable to use a loss function that allows the estimation of a failure probability of zero. A positive loss at the origin allows the estimation of zero and in risk analyses estimating a zero failure probability simply means that no risk is expected for further detail see Norstrom (1996). Three loss functions are used to obtain the Bayes estimators along with posterior risks, i.e., the squared error (SE) loss function, weighted squared error (WSE) loss function and quadratic (Q) loss function. The most commonly used loss function is (SE) loss function defined by $L_{1}=l\left(\hat{\theta}_{S E}-\theta\right)=\left(\hat{\theta}_{S E}-\theta\right)^{2}$, where $\hat{\theta}_{S E}$ is a decision rule to estimate parameter $\theta$. The Bayes estimator under SE loss function is $\hat{\theta}_{S E}=E(\theta)$ and posterior risk under $\mathrm{SE}$ loss function is $\rho\left(\hat{\theta}_{S E}\right)=E\left(\theta^{2} \mid \mathrm{x}\right)-\{E(\theta \mid \mathrm{x})\}^{2}$. The weighted squared error (WSE) loss function which is 
of concern is $L_{2}=\theta^{-1}\left(\theta-\hat{\theta}_{W S E}\right)^{2}$, the Bayes estimator under WSE loss function is $\hat{\theta}_{W S E}=\left\{E\left(\theta^{-1} \mid \mathrm{x}\right)\right\}^{-1}$ and posterior risk under WSE loss function is $\rho\left(\hat{\theta}_{\text {WSE }}\right)=E(\theta \mid \mathrm{x})-\left\{E\left(\theta^{-1} \mid \mathrm{x}\right)\right\}^{-1}$. The third loss function is the quadratic loss function which is defined as $L_{3}=\left(1-\theta^{-1} \hat{\theta}_{Q}\right)^{2}$. The Bayes estimator and posterior risk under $\mathrm{Q}$ loss function are $\hat{\theta}_{Q}=\left\{E\left(\theta^{-2} \mid \mathrm{x}\right)\right\}^{-1} E\left(\theta^{-1} \mid \mathrm{x}\right), \rho\left(\hat{\theta}_{Q}\right)=1-\left\{E\left(\theta^{-2} \mid \mathrm{x}\right)\right\}^{-1}\left\{E\left(\theta^{-1} \mid \mathrm{x}\right)\right\}^{2}$.

Where $E$ denotes the expectation with respect to the posterior distribution of $\theta$. Thus the posterior expectation of any function of parameter, say $U\left(\lambda_{1}, \lambda_{2}, p_{1}\right)$ can be written as:

$\hat{U}\left(\lambda_{1}, \lambda_{2}, p_{1}\right)=E\left\{U\left(\lambda_{1}, \lambda_{2}, p_{1}\right) \mid\left(\mathrm{x}, \eta_{i}\right)\right\}=\frac{\int_{0}^{\infty} \int_{0}^{\infty} \int_{0}^{1} U\left(\lambda_{1}, \lambda_{2}, p_{1}\right) g\left(\lambda_{1}, \lambda_{1}, p_{1} \mid \mathrm{x}\right) d p_{1} d \lambda_{1} d \lambda_{2}}{\int_{0}^{\infty} \int_{0}^{\infty} \int_{0}^{1} g\left(\lambda_{1}, \lambda_{1}, p_{1} \mid \mathrm{x}\right) d p_{1} d \lambda_{1} d \lambda_{2}}$.

However, it is not possible to evaluate (5) in closed-form. We obtain the Bayes estimates using the curvature method.

\section{Simulation study}

In this section, we perform a Monte Carlo simulation to observe the behavior of the proposed estimator of the parameters and respective posterior risks for different sample sizes, for different priors, for different parametric values $\left(\lambda_{1}, \lambda_{2}\right) \in(3,5),(0.5,1.5),(4,2), T=10, p_{1} \in 0.4, \quad$ fixing $\quad \eta_{1}=\eta_{2}=0.5$, and $\eta_{1}=0.25, \eta_{2}=0.5$. Samples of size $n=30,60$ and 100 were generated from the two component mixture of the shifted Gompertz distribution. A well-known procedure in simulation for computer generation of random variables is the inverse transform method. This method provides the most straightforward procedure to generate samples of a given distribution when its quantile function exists in closed-form. The quantile function of the shifted Gompertz distribution can be expressed in closed-form in terms of the Lambert W function. As a consequence, simulation studies involving the shifted Gompertz distribution can easily be carried out for theoretical and practical purposes. From a computational point of view, since the Lambert $\mathrm{W}$ function is efficiently implemented in various computer algebra systems Matlab and Mathematica. Therefore, the inverse transform method can directly be applied to generate samples of the shifted Gompertz distribution. Probabilistic mixing is used to generate the mixture data. To generate the mixture model, a random number ' $u$ ' is generated from the uniform distribution on $(0,1)$. If $u<p_{1}$ the observation is taken randomly from $F_{1}$ (the shifted Gompertz distribution with parameter $\lambda_{1}$ ) and If $u>p_{1}$ the observation is taken randomly from $F_{2}$ (the shifted Gompertz distribution with parameter $\left.\lambda_{2}\right)$. The values of hyperparameters $\left(a_{1}, b_{1}, a_{2}, b_{2}\right)$ have been selected in such a manner that the prior mean becomes the expected value of the corresponding parameter. The hyperparameters consider in the simulation study are 
$(6,2,10,2),(1,2,3,2)$ and $(8,2,4,2)$. All observations that exceeded $T$ were treated as censored. For each of the combinations of parameters, sample sizes, we generated 1000 samples using Mathematica. For each of 1000 samples, the Bayes estimates and posterior risks are not in closed form and calculated by using the curvature integration method. There are two main reasons for one to need to do numerical integration: analytical integration may be impossible or infeasible, or one wishes to integrate tabulated data rather than known functions. In general, we derive numerical integration methods by splitting the interval $[a, b]$ into small subintervals, approximate $f$ by a polynomial on each subinterval, integrate this polynomial rather than $f$, and then add together the contributions from each subinterval. This is the strategy we follow for deriving numerical integration methods, and this works as long as $f$ can be approximated well by polynomials on each subinterval. The average of these estimates and corresponding posterior risks are reported in Table 2-7.

Table 2: Bayes estimates and their posterior risks in parentheses under SE loss function for different parametric points when $\eta_{1}=\eta_{2}=0.5$.

\begin{tabular}{|c|c|c|c|c|c|c|}
\hline \multirow{2}{*}{$n$} & \multicolumn{3}{|c|}{ Gamma prior } & \multicolumn{3}{|c|}{ Uniform prior } \\
\hline & $\hat{\lambda}_{1}=3$ & $\hat{\lambda}_{2}=5$ & $\hat{p}_{1}=0.4$ & $\hat{\lambda}_{1}=3$ & $\hat{\lambda}_{2}=5$ & $\hat{p}_{1}=0.4$ \\
\hline 30 & $\begin{array}{l}3.79007 \\
(0.409413)\end{array}$ & $\begin{array}{l}4.20046 \\
(0.326908)\end{array}$ & $\begin{array}{l}0.40625 \\
(0.007309)\end{array}$ & $\begin{array}{l}2.80835 \\
(0.618781)\end{array}$ & $\begin{array}{l}3.77180 \\
(0.502621)\end{array}$ & $\begin{array}{l}0.42151 \\
(0.007527)\end{array}$ \\
\hline 60 & $\begin{array}{l}3.27266 \\
(0.303047)\end{array}$ & $\begin{array}{l}4.25863 \\
(0.244741)\end{array}$ & $\begin{array}{l}0.40322 \\
(0.003819)\end{array}$ & $\begin{array}{l}2.86440 \\
(0.344531)\end{array}$ & $\begin{array}{l}3.89331 \\
(0.329380)\end{array}$ & $\begin{array}{l}0.41739 \\
(0.003939)\end{array}$ \\
\hline 100 & $\begin{array}{l}2.99339 \\
(0.187709)\end{array}$ & $\begin{array}{l}4.58252 \\
(0.193125)\end{array}$ & $\begin{array}{l}0.40196 \\
(0.002333)\end{array}$ & $\begin{array}{l}2.90215 \\
(0.213533)\end{array}$ & $\begin{array}{l}4.47016 \\
(0.247248) \\
\end{array}$ & $\begin{array}{l}0.39661 \\
(0.002419)\end{array}$ \\
\hline$n$ & $\hat{\lambda}_{1}=0.5$ & $\hat{\lambda}_{2}=1.5$ & $\hat{p}_{1}=0.4$ & $\hat{\lambda}_{1}=0.5$ & $\hat{\lambda}_{2}=1.5$ & $\hat{p}_{1}=0.4$ \\
\hline 30 & $\begin{array}{l}0.579411 \\
(0.014658)\end{array}$ & $\begin{array}{l}1.45757 \\
(0.105432)\end{array}$ & $\begin{array}{l}0.406228 \\
(0.007309)\end{array}$ & $\begin{array}{l}0.88059 \\
(0.220906)\end{array}$ & $\begin{array}{l}1.15438 \\
(0.167504)\end{array}$ & $\begin{array}{l}0.32182 \\
(0.012136)\end{array}$ \\
\hline 60 & $\begin{array}{l}0.52271 \\
(0.001873)\end{array}$ & $\begin{array}{l}1.45788 \\
(0.046582)\end{array}$ & $\begin{array}{l}0.40319 \\
(0.003820)\end{array}$ & $\begin{array}{l}0.647511 \\
(0.002141)\end{array}$ & $\begin{array}{l}1.25820 \\
(0.137583)\end{array}$ & $\begin{array}{l}0.35911 \\
(0.010937)\end{array}$ \\
\hline 100 & $\begin{array}{l}0.50034 \\
(0.000086)\end{array}$ & $\begin{array}{l}1.45814 \\
(0.023931)\end{array}$ & $\begin{array}{l}0.40195 \\
(0.002313)\end{array}$ & $\begin{array}{l}0.50212 \\
(0.001102)\end{array}$ & $\begin{array}{l}1.43859 \\
(0.024999)\end{array}$ & $\begin{array}{l}0.40194 \\
(0.002334)\end{array}$ \\
\hline$n$ & $\hat{\lambda}_{1}=4$ & $\hat{\lambda}_{2}=2$ & & $\hat{\lambda}_{1}=4$ & $\hat{\lambda}_{2}=2$ & $\hat{p}_{1}=0.4$ \\
\hline 30 & $\begin{array}{l}4.27034 \\
(0.328570) \\
\end{array}$ & $\begin{array}{l}2.16131 \\
(0.122003) \\
\end{array}$ & $\begin{array}{l}0.40625 \\
(0.007305) \\
\end{array}$ & $\begin{array}{l}3.34015 \\
(0.600769) \\
\end{array}$ & $\begin{array}{l}1.73905 \\
(0.162738) \\
\end{array}$ & $\begin{array}{l}0.39063 \\
(0.007309) \\
\end{array}$ \\
\hline 60 & $\begin{array}{l}4.12458 \\
(0.281236)\end{array}$ & $\begin{array}{l}2.06151 \\
(0.0867007)\end{array}$ & $\begin{array}{l}0.403226 \\
(0.003816)\end{array}$ & $\begin{array}{l}3.52803 \\
(0.391835) \\
\end{array}$ & $\begin{array}{l}1.78048 \\
(0.087179)\end{array}$ & $\begin{array}{l}0.40323 \\
(0.003820)\end{array}$ \\
\hline 00 & $\begin{array}{l}3.98141 \\
(0.240661)\end{array}$ & $\begin{array}{l}1.97365 \\
(0.044765)\end{array}$ & $\begin{array}{l}0.401961 \\
(0.002236)\end{array}$ & $\begin{array}{l}3.58380 \\
(0.241341)\end{array}$ & $\begin{array}{l}1.86515 \\
(0.045346)\end{array}$ & $\begin{array}{l}0.40196 \\
(0.002338)\end{array}$ \\
\hline
\end{tabular}


Table 3: Bayes estimates and their posterior risks in parentheses under SE loss function for different parametric points when $\eta_{1}=0.25, \eta_{2}=0.5$.

\begin{tabular}{|c|l|l|l|l|l|l|}
\hline \multirow{2}{*}{$n$} & \multicolumn{3}{|c|}{ Gamma prior } & \multicolumn{3}{c|}{ Uniform prior } \\
\cline { 2 - 7 } & \multicolumn{1}{|c|}{$\hat{\lambda}_{1}=3$} & \multicolumn{1}{|c|}{$\hat{\lambda}_{2}=5$} & $\hat{p}_{1}=0.4$ & \multicolumn{1}{|c|}{$\hat{\lambda}_{1}=3$} & \multicolumn{1}{|c|}{$\hat{\lambda}_{2}=5$} & $\hat{p}_{1}=0.4$ \\
\hline 30 & 3.56934 & 3.89195 & 0.40625 & 3.15419 & 3.88102 & 0.40725 \\
& $(0.456082)$ & $(0.431195)$ & $(0.007084)$ & $(0.561008)$ & $(0.438071)$ & $(0.007309)$ \\
\hline 60 & 3.40623 & 4.47885 & 0.40323 & 2.99863 & 4.02775 & 0.40383 \\
& $(0.307405)$ & $(0.177566)$ & $(0.003719)$ & $(0.330063)$ & $(0.282345)$ & $(0.003820)$ \\
\hline \multirow{2}{*}{100} & 3.23124 & 4.49654 & 0.40166 & 2.98159 & 4.59987 & 0.401961 \\
& $(0.215864)$ & $(0.147134)$ & $(0.002334)$ & $(0.22273)$ & $(0.205389)$ & $(0.002334)$ \\
\hline \multirow{2}{*}{$n$} & $\hat{\lambda}_{1}=0.5$ & $\hat{\lambda}_{2}=1.5$ & $\hat{p}_{1}=0.4$ & $\hat{\lambda}_{1}=0.5$ & $\hat{\lambda}_{2}=1.5$ & $\hat{p}_{1}=0.4$ \\
\hline \multirow{2}{*}{30} & 0.602047 & 1.45492 & 0.40614 & 0.61200 & 1.37675 & 0.40625 \\
& $(0.018955)$ & $(0.105755)$ & $(0.007312)$ & $(0.020280)$ & $(0.106368)$ & $(0.007094)$ \\
\hline \multirow{2}{*}{60} & 0.15929 & 1.45727 & 0.40321 & 0.53996 & 1.38554 & 0.40320 \\
& $(0.003965)$ & $(0.046027)$ & $(0.003816)$ & $(0.006167)$ & $(0.047845)$ & $(0.003820)$ \\
\hline \multirow{2}{*}{100} & 0.50819 & 1.45952 & 0.40195 & 0.509279 & 1.43939 & 0.40295 \\
& $(0.001375)$ & $(0.026456)$ & $(0.002321)$ & $(0.001485)$ & $(0.028926)$ & $(0.002340)$ \\
\hline \multirow{2}{*}{$n$} & $\hat{\lambda}_{1}=4$ & $\hat{\lambda}_{2}=2$ & $\hat{p}_{1}=0.4$ & $\hat{\lambda}_{1}=4$ & $\hat{\lambda}_{2}=2$ & $\hat{p}_{1}=0.4$ \\
\hline \multirow{2}{*}{30} & 4.34363 & 2.04862 & 0.40625 & 3.57312 & 1.78059 & 0.40664 \\
& $(0.289527)$ & $(0.196335)$ & $(0.007210)$ & $(0.563268)$ & $(0.198252)$ & $(0.007408)$ \\
\hline \multirow{2}{*}{60} & 4.18177 & 1.99819 & 0.40323 & 3.58931 & 1.79526 & 0.40332 \\
& $(0.284460)$ & $(0.095608)$ & $(0.00376)$ & $(0.375443)$ & $(0.096505)$ & $(0.003820)$ \\
\hline \multirow{2}{*}{100} & 4.04812 & 1.99978 & 0.40196 & 3.60573 & 1.86526 & 0.40198 \\
& $(0.223180)$ & $(0.043186)$ & $(0.002218)$ & $(0.269684)$ & $(0.045229)$ & $(0.002334)$ \\
\hline
\end{tabular}

Table 4: Bayes estimates and their posterior risks in parentheses under WSE loss function for different parametric points when $\eta_{1}=\eta_{2}=0.5$.

\begin{tabular}{|c|c|c|c|c|c|c|}
\hline \multirow{2}{*}{$n$} & \multicolumn{3}{|c|}{ Gamma prior } & \multicolumn{3}{c|}{ Uniform prior } \\
\cline { 2 - 7 } & $\hat{\lambda}_{1}=3$ & $\hat{\lambda}_{2}=5$ & $\hat{p}_{1}=0.4$ & $\hat{\lambda}_{1}=3$ & $\hat{\lambda}_{2}=5$ & $\hat{p}_{1}=0.4$ \\
\hline \multirow{2}{*}{30} & 2.69237 & 3.91390 & 0.38803 & 2.47605 & 3.86169 & 0.38709 \\
& $(0.149124)$ & $(0.094741)$ & $(0.019153)$ & $(0.179939)$ & $(0.117508)$ & $(0.019153)$ \\
\hline \multirow{2}{*}{60} & 2.75664 & 4.06623 & 0.393443 & 2.58039 & 3.98539 & 0.39343 \\
& $(0.088179)$ & $(0.062029)$ & $(0.009783)$ & $(0.104887)$ & $(0.081891)$ & $(0.009783)$ \\
\hline \multirow{2}{*}{100} & 2.84701 & 4.50663 & 0.39604 & 2.70179 & 4.48981 & 0.39604 \\
& $(0.058691)$ & $(0.047206)$ & $(0.005911)$ & $(0.066727)$ & $(0.053517)$ & $(0.005922)$ \\
\hline \multirow{2}{*}{$n$} & $\hat{\lambda}_{1}=0.5$ & $\hat{\lambda}_{2}=1.5$ & $\hat{p}_{1}=0.4$ & $\hat{\lambda}_{1}=0.5$ & $\hat{\lambda}_{2}=1.5$ & $\hat{p}_{1}=0.4$ \\
\hline \multirow{2}{*}{30} & 0.53203 & 1.29272 & 0.38702 & 0.53048 & 1.28193 & 0.38752 \\
& $(0.011165)$ & $(0.065209)$ & $(0.019162)$ & $(0.011313)$ & $(0.071869)$ & $(0.019164)$ \\
\hline \multirow{2}{*}{60} & 0.51566 & 1.39578 & 0.39342 & 0.51308 & 1.37321 & 0.39341 \\
& $(0.002101)$ & $(0.032878)$ & $(0.009784)$ & $(0.002445)$ & $(0.033773)$ & $(0.009785)$ \\
\hline \multirow{2}{*}{100} & 0.50711 & 1.42375 & 0.39602 & 0.50154 & 1.42239 & 0.39603 \\
& $(0.002953)$ & $(0.019553)$ & $(0.00590)$ & $(0.001684)$ & $(0.020558)$ & $(0.005913)$ \\
\hline \multirow{2}{*}{$n$} & $\hat{\lambda}_{1}=4$ & $\hat{\lambda}_{2}=2$ & $\hat{p}_{1}=0.4$ & $\hat{\lambda}_{1}=4$ & $\hat{\lambda}_{2}=2$ & $\hat{p}_{1}=0.4$ \\
\hline \multirow{2}{*}{30} & 3.45814 & 1.70583 & 0.38710 & 3.27966 & 1.57392 & 0.38709 \\
& $(0.138723)$ & $(0.081224)$ & $(0.019153)$ & $(0.195983)$ & $(0.087437)$ & $(0.019153)$ \\
\hline \multirow{2}{*}{60} & 3.62881 & 1.726314 & 0.39343 & 3.39822 & 1.66235 & 0.39344 \\
& $(0.095503)$ & $(0.041621)$ & $(0.009683)$ & $(0.116526)$ & $(0.046169)$ & $(0.009731)$ \\
\hline \multirow{2}{*}{100} & 3.77451 & 1.86758 & 0.39560 & 3.43749 & 1.76372 & 0.39604 \\
& $(0.069352)$ & $(0.026601)$ & $(0.005810)$ & $(0.077571)$ & $(0.027288)$ & $(0.005921)$ \\
\hline
\end{tabular}


Table 5: Bayes estimates and their posterior risks in parentheses under WSE loss function for different parametric points when $\eta_{1}=0.25, \eta_{2}=0.5$.

\begin{tabular}{|c|c|c|c|c|c|c|}
\hline \multirow{2}{*}{$n$} & \multicolumn{3}{|c|}{ Gamma prior } & \multicolumn{3}{c|}{ Uniform prior } \\
\cline { 2 - 7 } & $\hat{\lambda}_{1}=3$ & $\hat{\lambda}_{2}=5$ & $\hat{p}_{1}=0.4$ & $\hat{\lambda}_{1}=3$ & $\hat{\lambda}_{2}=5$ & $\hat{p}_{1}=0.4$ \\
\hline \multirow{2}{*}{30} & 2.70909 & 4.01021 & 0.38709 & 2.87543 & 3.87434 & 0.38807 \\
& $(0.151209)$ & $(0.091507)$ & $(0.019152)$ & $(0.195701)$ & $(0.118974)$ & $(0.019053)$ \\
\hline \multirow{2}{*}{60} & 2.77838 & 4.06322 & 0.39344 & 2.89951 & 3.98871 & 0.39934 \\
& $(0.092770)$ & $(0.062121)$ & $(0.009783)$ & $(0.112231)$ & $(0.074917)$ & $(0.009832)$ \\
\hline \multirow{2}{*}{100} & 2.86267 & 4.46404 & 0.39604 & 2.86434 & 4.42077 & 0.39857 \\
& $(0.058317)$ & $(0.046317)$ & $(0.005921)$ & $(0.065738)$ & $(0.050219)$ & $(0.005938)$ \\
\hline \multirow{2}{*}{$n$} & $\hat{\lambda}_{1}=0.5$ & $\hat{\lambda}_{2}=1.5$ & $\hat{p}_{1}=0.4$ & $\hat{\lambda}_{1}=0.5$ & $\hat{\lambda}_{2}=1.5$ & $\hat{p}_{1}=0.4$ \\
\hline \multirow{2}{*}{30} & 0.52834 & 1.29446 & 0.38709 & 0.53086 & 1.27331 & 0.39604 \\
& $(0.010902)$ & $(0.064789)$ & $(0.019154)$ & $(0.024083)$ & $(0.071065)$ & $(0.019373)$ \\
\hline \multirow{2}{*}{60} & 0.510827 & 1.39097 & 0.39343 & 0.52268 & 1.37969 & 0.395433 \\
& $(0.003957)$ & $(0.031994)$ & $(0.009784)$ & $(0.006446)$ & $(0.033193)$ & $(0.009788)$ \\
\hline \multirow{2}{*}{100} & 0.509483 & 1.43961 & 0.39604 & 0.50997 & 1.43960 & 0.39861 \\
& $(0.002049)$ & $(0.018745)$ & $(0.005923)$ & $(0.002857)$ & $(0.020665)$ & $(0.006219)$ \\
\hline \multirow{2}{*}{$n$} & $\hat{\lambda}_{1}=4$ & $\hat{\lambda}_{2}=2$ & $\hat{p}_{1}=0.4$ & $\hat{\lambda}_{1}=4$ & $\hat{\lambda}_{2}=2$ & $\hat{p}_{1}=0.4$ \\
\hline \multirow{2}{*}{30} & 3.50845 & 1.74355 & 0.38709 & 3.37434 & 1.63686 & 0.39781 \\
& $(0.136470)$ & $(0.083019)$ & $(0.019153)$ & $(0.185908)$ & $(0.09098)$ & $(0.021532)$ \\
\hline \multirow{2}{*}{60} & 3.60998 & 1.76874 & 0.39343 & 3.49503 & 1.68515 & 0.39843 \\
& $(0.096461)$ & $(0.043264)$ & $(0.009783)$ & $(0.111923)$ & $(0.044033)$ & $(0.009789)$ \\
\hline \multirow{2}{*}{100} & 3.74249 & 1.85287 & 0.39604 & 3.53686 & 1.86178 & 0.39960 \\
& $(0.068667)$ & $(0.026226)$ & $(0.005921)$ & $(0.079767)$ & $(0.026951)$ & $(0.006122)$ \\
\hline
\end{tabular}

Table 6: Bayes estimates and their posterior risks in parentheses under $Q$ loss function for different parametric points when $\eta_{1}=\eta_{2}=0.5$.

\begin{tabular}{|c|l|l|l|l|l|l|}
\hline \multirow{2}{*}{$n$} & \multicolumn{3}{|c|}{ Gamma prior } & \multicolumn{3}{c|}{ Uniform prior } \\
\cline { 2 - 7 } & \multicolumn{1}{|c|}{$\hat{\lambda}_{1}=3$} & \multicolumn{1}{|c|}{$\hat{\lambda}_{2}=5$} & $\hat{p}_{1}=0.4$ & \multicolumn{1}{|c|}{$\hat{\lambda}_{1}=3$} & \multicolumn{1}{c|}{$\hat{\lambda}_{2}=5$} & $\hat{p}_{1}=0.4$ \\
\hline 30 & 3.30204 & 4.30476 & 0.36867 & 2.23871 & 3.60019 & 0.36667 \\
& $(0.045433)$ & $(0.010684)$ & $(0.052078)$ & $(0.079977)$ & $(0.039114)$ & $(0.052778)$ \\
\hline 60 & 3.17656 & 4.40314 & 0.38321 & 2.37165 & 3.84865 & 0.38333 \\
& $(0.033626)$ & $(0.010522)$ & $(0.025674)$ & $(0.041454)$ & $(0.020883)$ & $(0.025694)$ \\
\hline \multirow{2}{*}{100} & 2.96714 & 4.52941 & 0.39157 & 2.41415 & 4.45692 & 0.39231 \\
& $(0.022206)$ & $(0.009431)$ & $(0.015131)$ & $(0.024964)$ & $(0.013723)$ & $(0.015250)$ \\
\hline \multirow{2}{*}{$n$} & $\hat{\lambda}_{1}=0.5$ & $\hat{\lambda}_{2}=1.5$ & $\hat{p}_{1}=0.4$ & $\hat{\lambda}_{1}=0.5$ & $\hat{\lambda}_{2}=1.5$ & $\hat{p}_{1}=0.4$ \\
\hline \multirow{2}{*}{30} & 0.53201 & 1.39847 & 0.366567 & 0.53010 & 1.18985 & 0.366469 \\
& $(0.015955)$ & $(0.050641)$ & $(0.052819)$ & $(0.017578)$ & $(0.056442)$ & $(0.052858)$ \\
\hline \multirow{2}{*}{60} & 0.51446 & 1.39975 & 0.38314 & 0.51249 & 1.23406 & 0.383307 \\
& $(0.005894)$ & $(0.026326)$ & $(0.025695)$ & $(0.006422)$ & $(0.002795)$ & $(0.025701)$ \\
\hline \multirow{2}{*}{100} & 0.50183 & 1.45074 & 0.38999 & 0.50114 & 1.39562 & 0.38998 \\
& $(0.001007)$ & $(0.014332)$ & $(0.015250)$ & $(0.001021)$ & $(0.016765)$ & $(0.015253)$ \\
\hline \multirow{2}{*}{$n$} & $\hat{\lambda}_{1}=4$ & $\hat{\lambda}_{2}=2$ & $\hat{p}_{1}=0.4$ & $\hat{\lambda}_{1}=4$ & $\hat{\lambda}_{2}=2$ & $\hat{p}_{1}=0.4$ \\
\hline \multirow{2}{*}{30} & 4.13342 & 1.80924 & 0.36679 & 2.96955 & 1.61289 & 0.36667 \\
& $(0.023352)$ & $(0.047586)$ & $(0.052763)$ & $(0.066981)$ & $(0.055547)$ & $(0.052778)$ \\
\hline \multirow{2}{*}{60} & 3.95647 & 1.84806 & 0.38325 & 3.21052 & 1.65984 & 0.38333 \\
& $(0.021646)$ & $(0.025636)$ & $(0.025541)$ & $(0.037276)$ & $(0.027778)$ & $(0.025694)$ \\
\hline \multirow{2}{*}{100} & 3.99512 & 1.87472 & 0.38923 & 3.52063 & 1.761403 & 0.39145 \\
& $(0.016714)$ & $(0.015871)$ & $(0.015190)$ & $(0.023844)$ & $(0.016676)$ & $(0.015250)$ \\
\hline
\end{tabular}


Table 7: Bayes estimates and their posterior risks in parentheses under $Q$ loss function for different parametric points when $\eta_{1}=0.25, \eta_{2}=0.5$.

\begin{tabular}{|c|c|c|c|c|c|c|}
\hline \multirow{2}{*}{$n$} & \multicolumn{3}{|c|}{ Gamma prior } & \multicolumn{3}{|c|}{ Uniform prior } \\
\hline & $\hat{\lambda}_{1}=3$ & $\hat{\lambda}_{2}=5$ & $\hat{p}_{1}=0.4$ & $\hat{\lambda}_{1}=3$ & $\hat{\lambda}_{2}=5$ & $\hat{p}_{1}=0.4$ \\
\hline 30 & $\begin{array}{c}3.52761 \\
(0.041418)\end{array}$ & $\begin{array}{c}4.52392 \\
(0.010206)\end{array}$ & $\begin{array}{c}0.36667 \\
(0.052778)\end{array}$ & $\begin{array}{c}2.55618 \\
(0.076166)\end{array}$ & $\begin{array}{c}3.60576 \\
(0.038598)\end{array}$ & $\begin{array}{c}0.365807 \\
(0.054873)\end{array}$ \\
\hline 60 & $\begin{array}{c}3.20083 \\
(0.031351)\end{array}$ & $\begin{array}{c}4.54391 \\
(0.009834)\end{array}$ & $\begin{array}{c}0.38333 \\
(0.025674)\end{array}$ & $\begin{array}{c}2.74051 \\
(0.040217)\end{array}$ & $\begin{array}{c}3.89762 \\
(0.020081)\end{array}$ & $\begin{array}{c}0.38273 \\
(0.025694)\end{array}$ \\
\hline 100 & $\begin{array}{c}3.02593 \\
(0.021865) \\
\end{array}$ & $\begin{array}{c}4.54922 \\
(0.008430)\end{array}$ & $\begin{array}{c}0.39125 \\
(0.01525) \\
\end{array}$ & $\begin{array}{c}2.80950 \\
(0.024698)\end{array}$ & $\begin{array}{c}4.53158 \\
(0.013433) \\
\end{array}$ & $\begin{array}{c}0.39087 \\
(0.015682) \\
\end{array}$ \\
\hline$n$ & $\hat{\lambda}_{1}=0.5$ & $\hat{\lambda}_{2}=1.5$ & $\hat{p}_{1}=0.4$ & $\hat{\lambda}_{1}=0.5$ & $\hat{\lambda}_{2}=1.5$ & $\hat{p}_{1}=0.4$ \\
\hline 30 & $\begin{array}{c}0.54364 \\
(0.023970)\end{array}$ & $\begin{array}{c}1.36568 \\
(0.050739)\end{array}$ & $\begin{array}{c}0.366471 \\
(0.052857)\end{array}$ & $\begin{array}{c}0.52408 \\
(0.025407)\end{array}$ & $\begin{array}{c}1.21533 \\
(0.057450)\end{array}$ & $\begin{array}{c}0.36407 \\
(0.052889)\end{array}$ \\
\hline 60 & $\begin{array}{c}0.51029 \\
(0.007165)\end{array}$ & $\begin{array}{c}1.39505 \\
(0.026574)\end{array}$ & $\begin{array}{c}0.38236 \\
(0.025709)\end{array}$ & $\begin{array}{c}0.50929 \\
(0.007637)\end{array}$ & $\begin{array}{c}1.29512 \\
(0.027854)\end{array}$ & $\begin{array}{c}0.38137 \\
(0.026937)\end{array}$ \\
\hline 100 & $\begin{array}{c}0.50953 \\
(0.004979) \\
\end{array}$ & $\begin{array}{c}1.44519 \\
(0.016117) \\
\end{array}$ & $\begin{array}{c}0.38999 \\
(0.015251) \\
\end{array}$ & $\begin{array}{c}0.50385 \\
(0.006023)\end{array}$ & $\begin{array}{c}1.38673 \\
(0.016797) \\
\end{array}$ & $\begin{array}{c}0.39816 \\
(0.015469) \\
\end{array}$ \\
\hline$n$ & $\hat{\lambda}_{1}=4$ & $\hat{\lambda}_{2}=2$ & $\hat{p}_{1}=0.4$ & $\hat{\lambda}_{1}=4$ & $\hat{\lambda}_{2}=2$ & $\hat{p}_{1}=0.4$ \\
\hline 30 & $\begin{array}{c}4.24280 \\
(0.020152)\end{array}$ & $\begin{array}{c}1.93463 \\
(0.047479)\end{array}$ & $\begin{array}{c}0.36667 \\
(0.052778)\end{array}$ & $\begin{array}{c}3.17187 \\
(0.063645)\end{array}$ & $\begin{array}{c}1.69135 \\
(0.055505)\end{array}$ & $\begin{array}{c}0.36635 \\
(0.053678)\end{array}$ \\
\hline 60 & $\begin{array}{c}4.21140 \\
(0.016740)\end{array}$ & $\begin{array}{c}1.96996 \\
(0.025642)\end{array}$ & $\begin{array}{c}0.38343 \\
(0.025694)\end{array}$ & $\begin{array}{c}3.39504 \\
(0.034875)\end{array}$ & $\begin{array}{c}1.85113 \\
(0.027775)\end{array}$ & $\begin{array}{c}0.38453 \\
(0.02643)\end{array}$ \\
\hline 100 & $\begin{array}{c}4.00304 \\
(0.015807)\end{array}$ & $\begin{array}{c}1.99883 \\
(0.015873)\end{array}$ & $\begin{array}{c}0.39217 \\
(0.015253)\end{array}$ & $\begin{array}{c}3.56545 \\
(0.023082)\end{array}$ & $\begin{array}{c}1.861635 \\
(0.016662)\end{array}$ & $\begin{array}{c}0.39142 \\
(0.016351)\end{array}$ \\
\hline
\end{tabular}

Based on Table 2-7, some results are very obvious. The foremost point that requires attention is that posterior risk decreases as we increase sample size and Bayesian estimates become very close to the true values of the parameters as we increase the sample size. The posterior risk of the Bayes estimator is a notable criterion for computing the performance of the different estimators. The amount of the posterior risk is directly proportional to true parametric values and is inversely proportional to the sample size.

It can be seen that Bayes estimators performed well under quadratic loss function than the squared error loss function and weighted squared error loss function. Bayes estimates are found to be under estimated under weighted squared error loss function based on all both priors. Bayes estimators are efficient under quadratic loss function; In fact the use of quadratic loss function unveiled the smallest posterior risk, which is really an advantageous property. Furthermore, we obtain efficient results using the gamma prior than the uniform prior. Posterior risks for the Bayes estimates assuming uniform prior is also little high. Hence, gamma prior has a clear edge over uniform prior. The selection of the best prior and loss function does not depend on sample sizes. Whereas, it is to be noted that selection of the best prior/loss function for a given loss function/prior is made on the basis of minimum posterior risks associated with it. Therefore we can easily make a selection of preferable prior and loss function. Effect of the changing in shape parameters $\eta_{i}$ from $\eta_{i}=0.5$ for $i=1,2$, to $\eta_{1}=0.25$ and $\eta_{1}=0.25$ yields some closer estimates to the true population parameter. 


\section{Bayesian Estimation (case b: all parameters are random)}

Now we study the most significant aspect when both shape and scale parameters are unknown. We use both informative and noninformative priors for the parameters $\lambda_{i}$ and $\eta_{i}$.

\subsection{Bayesian estimation using informative / noninformative prior}

It is supposed that $\lambda_{i}$ and $\eta_{i}$ each have independent gamma priors $\left(a_{i}, b_{i}\right)$ and gamma $\left(c_{i}\right.$, $d_{i}$ ) priors, respectively, where $i=1,2$ and parameters $p_{1}$ is assumed to have a uniform prior. The joint prior $\lambda_{i}, \eta_{i}$ and $p_{1}$ may take the form

$$
g\left(\lambda_{i}, \eta_{i}, p_{1} \mid a_{i}, b_{i}, c_{i}, d_{i}\right) \propto \lambda_{1}^{a_{1}-1} \exp \left(-b_{1} \lambda_{1}\right) \lambda_{2}{ }^{a_{2}-1} \exp \left(-b_{2} \lambda_{2}\right) \eta_{1}^{c_{1}-1} \exp \left(\eta_{1} d_{1}\right) \eta_{2}^{c_{2}-1} \exp \left(\eta_{2} d_{2}\right) .
$$

Then the joint posterior distribution for $\lambda_{i}, \eta_{i}$ and $p_{1}$ in this case is obtained by multiplying the joint prior and the likelihood function together as:

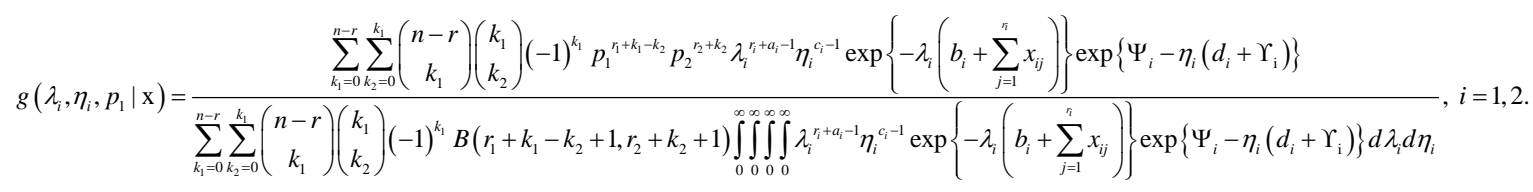

The posterior expectation of any function of parameter, say $U\left(\lambda_{i}, \eta_{i}, p_{1}\right)$ can be obtained as:

$\hat{U}\left(\lambda_{i}, \eta_{i}, p_{1}\right)=E\left\{U\left(\lambda_{i}, \eta_{i}, p_{1} \mid \mathrm{x}\right)\right\}=\frac{\int_{0}^{\infty} \int_{0}^{\infty} \int_{0}^{\infty} \int_{0}^{\infty} \int_{0}^{1} U\left(\lambda_{1}, \lambda_{2}, \eta_{1}, \eta_{2}, p_{1}\right) g\left(\lambda_{1}, \lambda_{2}, \eta_{1}, \eta_{2}, p_{1} \mid \mathrm{x}\right) d p_{1} d \lambda_{1} d \lambda_{2} d \eta_{1} d \eta_{2}}{\int_{0}^{\infty} \int_{0}^{\infty} \int_{0}^{\infty} \int_{0}^{\infty} \int_{0}^{1} g\left(\lambda_{1}, \lambda_{2}, \eta_{1}, \eta_{2}, p_{1} \mid \mathrm{x}\right) d p_{1} d \lambda_{1} d \lambda_{2} d \eta_{1} d \eta_{2}}$.

However, it is not possible to evaluate (6) in explicit tractable form. Hence such computational difficulties can be solved to obtain the Bayes estimates using the curvature method of integration in SAS or Mathematica. Bayes estimates under uniform prior can be obtained simply by equating the hyperparameters $a_{i}$ equal to one and $b_{i}$ equal to zero.

\subsection{Reliability function of shifted Gompertz mixture distribution}

The reliability function or survival function of shifted Gompertz mixture distribution is given by:

$$
R(t)=p_{1}\left(1-\left\{1-\exp \left(-\lambda_{1} T\right)\right\} \exp \left\{-\eta_{1} \exp \left(-\lambda_{1} T\right)\right\}\right)+p_{2}\left(1-\left\{1-\exp \left(-\lambda_{2} T\right)\right\} \exp \left\{-\eta_{2} \exp \left(-\lambda_{2} T\right)\right\}\right) .
$$

The Bayesian estimation of reliability function using different priors under different loss function is obtained as:

$$
\hat{R}\left(\lambda_{i}, \eta_{i}, p_{1} \mid t\right)=E\left\{R\left(\lambda_{i}, \eta_{i}, p_{1}\right) \mid(\mathrm{x}, t)\right\}=\frac{\int_{0}^{\infty} \int_{0}^{\infty} \int_{0}^{\infty} \int_{0}^{\infty} \int_{0}^{1}\left\{R\left(\lambda_{i}, \eta_{i}, p_{1}\right) \mid t\right\} g\left(\lambda_{i}, \eta_{i}, p_{1} \mid \mathrm{x}\right) d p_{1} d \lambda_{1} d \lambda_{2} d \eta_{1} d \eta_{2}}{\int_{0}^{\infty} \int_{0}^{\infty} \int_{0}^{\infty} \int_{0}^{\infty} \int_{0}^{1} g\left(\lambda_{i}, \eta_{i}, p_{1} \mid \mathrm{x}\right) d p_{1} d \lambda_{1} d \lambda_{2} d \eta_{1} d \eta_{2}}
$$


The expressions of the estimators show that an analytic comparison of these estimators is not possible. To compare the different estimators of the reliability function of the shifted Gompertz mixture distribution obtained in this paper, probability mixing random sample of various sample sizes are generated as the procedure defined in previous section. Bayesian reliability estimates $\hat{R}_{S E}(t), \hat{R}_{W S E}(t)$ and $\hat{R}_{Q}(t)$ are then computed at $t=0.3$. The true value of $R(t)=0.60318$ for a given vector $\left(\lambda_{1}=4, \lambda_{2}=2, \eta_{1}=0.5, \eta_{2}=1.5, p_{1}=0.4\right) \quad$ and $\quad R(t)=0.935048$ for $\quad$ vector $\left(\lambda_{1}=0.5, \lambda_{2}=1.5, \eta_{1}=3, \eta_{2}=2, p_{1}=0.4\right)$. The values of hyperparameters have been selected in such manner that the prior mean becomes the expected value of the corresponding parameter as explained earlier. For comparing the performance of different Bayes estimates of reliability function posterior risk of estimates is a significant criterion. The amount of posterior risk is inversely proportional to the sample size. From the numerical results given in the Table 8-10, we observed that an increase in sample size provides improved Bayes estimates. The effect of sample size and parametric values on the Bayes estimates is in the form of over estimation. However as sample size increases the amount of posterior risks of Bayes estimates of parameters decrease.

The Bayes estimates of reliability function under SE loss function have smaller posterior risk as compared to other. The Bayes estimates of reliability function under uniform prior are under estimated and are pretty good and efficient under gamma prior.

Table 8: Bayes estimates and their posterior risks in parentheses under SE loss functions for different parametric points

\begin{tabular}{|c|c|c|c|c|c|}
\hline \multirow{3}{*}{$n$} & \multicolumn{5}{|c|}{ Gamma prior } \\
\cline { 2 - 6 } & $\hat{\lambda}_{1}=3$ & $\hat{\lambda}_{2}=2$ & $\hat{\eta}_{1}=0.25$ & $\hat{\eta}_{2}=0.5$ & $\hat{p}_{1}=0.4$ \\
\hline \multirow{2}{*}{30} & 2.95898 & 1.82169 & 0.512343 & 0.517194 & 0.40909 \\
& $(0.554873)$ & $(0.187358)$ & $(0.006359)$ & $(0.008937)$ & $(0.015002)$ \\
\hline \multirow{2}{*}{60} & 2.981637 & 1.86835 & 0.350041 & 0.541021 & 0.432261 \\
& $(0.266232)$ & $(0.063473)$ & $(0.000207)$ & $(0.000070)$ & $(0.003849)$ \\
\hline \multirow{2}{*}{100} & 2.997638 & 1.899615 & 0.25531 & 0.50213 & 0.401961 \\
& $(0.167537)$ & $(0.037928)$ & $(0.000042)$ & $(0.000003)$ & $(0.002338)$ \\
\hline
\end{tabular}

Uniform prior

\begin{tabular}{|c|c|c|c|c|c|}
\hline$n$ & $\hat{\lambda}_{1}=3$ & $\hat{\lambda}_{2}=2$ & $\hat{\eta}_{1}=0.25$ & $\hat{\eta}_{2}=0.5$ & $\hat{p}_{1}=0.4$ \\
\hline \multirow{2}{*}{30} & 3.26132 & 1.80534 & 0.60732 & 0.55035 & 0.40909 \\
& $(0.683581)$ & $(0.290182)$ & $(0.068279)$ & $(0.029580)$ & $(0.010510)$ \\
\hline \multirow{2}{*}{60} & 3.11887 & 1.82645 & 0.40206 & 0.50032 & 0.40323 \\
& $(0.328235)$ & $(0.071096)$ & $(0.001036)$ & $(0.000162)$ & $(0.003820)$ \\
\hline \multirow{2}{*}{100} & 2.890104 & 1.88971 & 0.30435 & 0.50008 & 0.401961 \\
& $(0.193881)$ & $(0.046146)$ & $(0.000335)$ & $(0.000035)$ & $(0.003338)$ \\
\hline
\end{tabular}


Table 9: Bayes estimates and their posterior risks in parentheses under $Q$ loss functions for different parametric points

\begin{tabular}{|c|c|c|c|c|c|}
\hline \multirow{2}{*}{$n$} & \multicolumn{5}{|c|}{ Gamma prior } \\
\cline { 2 - 6 } & $\hat{\lambda}_{1}=3$ & $\hat{\lambda}_{2}=2$ & $\hat{\eta}_{1}=0.25$ & $\hat{\eta}_{2}=0.5$ & $\hat{p}_{1}=0.4$ \\
\hline \multirow{2}{*}{30} & 2.56625 & 1.73582 & 0.41762 & 0.56472 & 0.38621 \\
& $(0.103790)$ & $(0.032513)$ & $(0.011135)$ & $(0.002035)$ & $(0.06135)$ \\
\hline \multirow{2}{*}{60} & 2.83579 & 1.83497 & 0.35276 & 0.53762 & 0.39756 \\
& $(0.030022)$ & $(0.013427)$ & $(0.000167)$ & $(0.000126)$ & $(0.015643)$ \\
\hline \multirow{2}{*}{100} & 2.99587 & 1.93176 & 0.25432 & 0.50216 & 0.399752 \\
& $(0.003525)$ & $(0.007927)$ & $(0.000022)$ & $(0.000001)$ & $(0.003521)$ \\
\hline
\end{tabular}

\begin{tabular}{|c|c|c|c|c|c|}
\hline \multirow{2}{*}{$n$} & $\hat{\lambda}_{1}=3$ & $\hat{\lambda}_{2}=2$ & $\hat{\eta}_{1}=0.25$ & $\hat{\eta}_{2}=0.5$ & $\hat{p}_{1}=0.4$ \\
\hline \multirow{2}{*}{30} & 2.36355 & 1.60335 & 0.52751 & 0.55206 & 0.35214 \\
& $(0.109660)$ & $(0.082069)$ & $(0.053393)$ & $(0.023779)$ & $(0.081256)$ \\
\hline \multirow{2}{*}{60} & 2.71681 & 1.67344 & 0.49509 & 0.50986 & 0.38333 \\
& $(0.040082)$ & $(0.027479)$ & $(0.001024)$ & $(0.000154)$ & $(0.025694)$ \\
\hline \multirow{2}{*}{100} & 2.94915 & 1.83191 & 0.30056 & 0.50624 & 0.39837 \\
& $(0.026819)$ & $(0.016819)$ & $(0.000072)$ & $(0.000078)$ & $(0.015256)$ \\
\hline
\end{tabular}

Table 10: Bayes estimates of reliability function and posterior risks in parentheses based on SE, WSE and Q loss functions for different parametric points

\begin{tabular}{|c|l|l|l|l|}
\hline \multicolumn{5}{|c|}{ Squared Error Loss Function } \\
\hline$R(t)$ & \multicolumn{2}{|c|}{0.603183} & \multicolumn{2}{c|}{0.935048} \\
\hline$n$ & Gamma prior & Uniform prior & Gamma prior & Uniform prior \\
\hline \multirow{2}{*}{10} & $\begin{array}{l}0.608992 \\
(0.005123)\end{array}$ & $\begin{array}{l}0.635939 \\
(0.008174)\end{array}$ & $\begin{array}{l}0.917035 \\
(0.001234)\end{array}$ & $\begin{array}{l}0.889385 \\
(0.001871)\end{array}$ \\
\hline \multirow{2}{*}{20} & $\begin{array}{l}0.606242 \\
(0.003138)\end{array}$ & $\begin{array}{l}0.624087 \\
(0.004434)\end{array}$ & $\begin{array}{l}0.917348 \\
(0.000724)\end{array}$ & $\begin{array}{l}0.898660 \\
(0.000797)\end{array}$ \\
\hline \multirow{2}{*}{30} & $\begin{array}{l}0.603516 \\
(0.002404)\end{array}$ & $\begin{array}{l}0.605571 \\
(0.003046)\end{array}$ & $\begin{array}{l}0.931998 \\
(0.000543)\end{array}$ & $\begin{array}{l}0.908853 \\
(0.000552)\end{array}$ \\
\hline \multicolumn{5}{|c|}{ Weighted Squared Error Loss Function } \\
\hline$n$ & Gamma prior & Uniform prior & Gamma prior & Uniform prior \\
\hline \multirow{2}{*}{10} & $\begin{array}{l}0.616730 \\
(0.008355)\end{array}$ & $\begin{array}{l}0.650812 \\
(0.012866)\end{array}$ & $\begin{array}{l}0.940192 \\
(0.000728)\end{array}$ & $\begin{array}{l}0.881507 \\
(0.002559)\end{array}$ \\
\hline \multirow{2}{*}{20} & $\begin{array}{l}0.610396 \\
(0.004961)\end{array}$ & $\begin{array}{l}0.622832 \\
(0.006634)\end{array}$ & $\begin{array}{l}0.935489 \\
(0.000637)\end{array}$ & $\begin{array}{l}0.885928 \\
(0.000915)\end{array}$ \\
\hline \multirow{2}{*}{30} & $\begin{array}{l}0.608583 \\
(0.004034)\end{array}$ & $\begin{array}{l}0.614829 \\
(0.005119)\end{array}$ & $\begin{array}{l}0.935149 \\
(0.005611)\end{array}$ & $\begin{array}{l}0.889226 \\
(0.000578)\end{array}$ \\
\hline \multicolumn{5}{|c|}{ Quadratic Loss Function } \\
\hline$n$ & Gamma prior & Uniform prior & Gamma prior & Uniform prior \\
\hline \multirow{2}{*}{10} & $\begin{array}{l}\text { G.598371 } \\
(0.015699)\end{array}$ & $\begin{array}{l}0.603183 \\
(0.023931)\end{array}$ & $\begin{array}{l}0.922038 \\
(0.001363)\end{array}$ & $\begin{array}{l}0.886824 \\
(0.002174)\end{array}$ \\
\hline \multirow{2}{*}{20} & $\begin{array}{l}0.606838 \\
(0.007914)\end{array}$ & $\begin{array}{l}0.613572 \\
(0.010269)\end{array}$ & $\begin{array}{l}0.924689 \\
(0.000951)\end{array}$ & $\begin{array}{l}0.889368 \\
(0.0010934)\end{array}$ \\
\hline \multirow{2}{*}{30} & 0.603284 & 0.596604 & 0.934510 & 0.897589 \\
& $(0.006899)$ & $(0.008672)$ & $(0.000657)$ & $(0.000676)$ \\
\hline
\end{tabular}




\section{Concluding remarks}

In this study, we propose a mixture shifted Gompertz model of lifetime study under censoring sampling scheme. To judge the relative performance of the Bayes estimators and also to deal with the problems of selecting the priors and loss functions at different sample sizes. The analysis has been considered assuming the cases when shape parameter is known and when all parameters are unknown based on right censored data. We have discussed Bayesian estimation of reliability function of mixture shifted Gompertz distribution using three different loss functions under informative and noninformative priors. The capability of Bayesian analysis ensures us to perform a comprehensive study to address of selecting the suitable prior and desirable loss function for the mixture shifted Gompertz model. It is seen that the closed form expression for the Bayes estimators are not possible, we obtain the approximate Bayes estimates. The simulation study has revealed some interested results related to Bayes estimates of parameters and reliability function. The posterior risks of the estimates of the parameters appeared to be quite large with relatively large values of the parameters and vice versa. Though posterior risk of parameters decrease as the sample size increase in each case. To address the problem of selecting prior and loss function we have observed that the Bayes estimator of parameters perform best under Q loss function assuming gamma prior in both cases when shape parameters are known and in the case where all parameters are taken as random. In the case where all parameters are taken as random Bayes estimates obtained in this case show under estimation for scale parameters and over estimation for shape parameters. Bayes estimates of reliability function are more efficient and pretty good under SE loss function, assuming gamma prior than other two functions assuming uniform prior.

\section{Reference}

1. Arnold, B. C., and Press, S. J. (1983). Bayesian inference for Pareto population. Journal of Econometrics, 21, 287-306.

2. Bass, F.M. (1969). A new product growth model for consumer durables. Management science, 15(5), 215-227.

3. Bemmaor, A. C., Lee, J. (2002). The impact of heterogeneity and ill-conditioning on diffusion model parameter estimates. Marketing. Science, 21(2), 209-220.

4. Bemmaor, A.C. (1994). Modeling the diffusion of new durable goods: word-ofmouth effect versus consumer heterogeneity. In Gilles Laurent, Gary L. Lilien, \& Bernard Pras (Eds.), Research traditions in marketing, 201 - 213. Boston, MA7 Kluwer.

5. Bernardo, J. M. (1979). Reference posterior distribution for Bayesian inference with discussion. Journal of the Royal Statistical Society: Series B, 41, 113-147.

6. Ghosh, S. K. Ebrahimi, N. (2001). Bayesian analysis of the mixing function in a mixture of two exponential distributions. Tech. Rep. 2531, Institute of Statistics Mimeographs, North Carolina State University, North Carolina State University.

7. Ismail, A. A. (2010). Bayes estimation of Gompertz distribution parameters and acceleration factor under partially accelerated life tests with type-I censoring. Journal of Statistical Computation and Simulation, 80(11), 1253-1264. 
8. Ismail, A. A. (2011). Planning step-stress life tests with type-II censored Data. Scientific Research and Essays, 6(19), 4021-4028.

9. Jiménez, P. F. and Jodrá (2009). A note on the moments and computer generation of the shifted Gompertz distribution, Communications in Statistics Theory and Methods, 38, 75-89.

10. Kiani, K., Arasan, J., \& Midi, H. (2012). Interval estimations for parameters of Gompertz model with time-dependent covariate and right censored data. Sains Malaysiana, 41(4), 471-480.

11. Mendenhall, W. and Hadar, R. J. (1958). Estimation of parameters of mixed exponentially distributed failure time distributions from censored life test data. Biometrika, 45(3-4), 504-520.

12. Norstrom, J. G. (1996). The use of precautionary loss functions in risk analysis. IEEE Trans. Reliab, 45 (3), 400-403.

13. Saleem M. and Aslam M. (2008). Bayesian analysis of the two component mixture of the Rayleigh distribution assuming the Uniform and the Jeffreys prior from censored data, Journal of Applied Statistics, 16(4), 105-113.

14. Saleem, M., Aslam, M. and Economou, P. (2010). On the Bayesian analysis of the mixture of power function distribution using the complete and the censored sample, Journal of Applied Statistics, 37(1), 25-40.

15. Saracoglu, B., Kaya, M.F. \& Abd-Elfattah, A.M. (2009). Comparison of estimators for stress-strength reliability in the Gompertz case. Hacettepe Journal of Mathematics and Statistics, 38(3), 339-349.

16. Sindhu, T. N., Froze, N., and Aslam, M. (2014). Preference of Prior for Bayesian Analysis of the Mixed Burr Type X Distribution under Type I Censored Samples, Pak.j.stat.oper.res. X (1), 17-39.

17. Sindhu, T. N., Froze, N., and Aslam, M. (2014). Statistical Inference for Mixed Burr Type II distribution using a Bayesian Framework, International Journal of Statistics and Economics, 13(1), 90-107.

18. Sindhu, T. N., Navid F. Aslam, M. and A. Shafiq (2016). Bayesian Inference of Mixture of two Rayleigh Distributions: A New Look. Journal of Mathematics, 48(2)(2016), 49-64.

19. Sindhu, T. N., Riaz, M. Aslam, M. and Ahmed, Z. (2016). A Study of Cumulative Quantity Control Chart for a mixture of Rayleigh Model under Bayesian Framework. Revista Colombiana de Estadística, 39(2), 185-204.

20. Sultan, K. S., Ismail, M. A. and Al-Moisheer, A. S., (2007). Mixture of two inverse Weibull distributions: properties and estimation, Computational Statistics \& Data Analysis, 51, 5377-5387.

21. Torres, F. J. (2014). Estimation of parameters of the shifted Gompertz distribution using least squares, maximum likelihood and moments methods. Journal of Computational and Applied Mathematics 255, 867-877. 